\title{
Selective reporting - a half signalling load algorithm for distributed sensing
}

\author{
Atílio Gameiro ${ }^{1}$, Carlos Ribeiro ${ }^{2^{*}}$ and José Quaresma
}

\begin{abstract}
Spectrum sensing is a powerful tool of the cognitive cycle to help circumvent the apparent spectrum scarcity faced by wireless transmission systems. To overcome the challenging issues faced by the localized sensing, multiple cognitive radios can cooperate to explore the multiuser diversity and generate a more reliable decision on the presence of a signal in the frequencies of interest. In such a cooperative sensing scenario, a common reporting channel is needed for the transmission of the information of each element. As the number of elements that participate in the sensing operation increases, so does the bandwidth demanded for the reporting channel, quickly becoming the limiting factor in this scenario. To tackle the issue of reducing the sensing report overhead, this paper introduces a new cooperative sensing scheme that introduces silence periods in the reporting and, relying on information theory principles, explores the information present in these periods to reduce by $50 \%$ the sensing reporting overhead while maintaining the same performance of standard reporting schemes. Numerical and experimental results confirm the theoretical analysis and show the predicted reduction in reporting overhead and performance preservation.
\end{abstract}

Keywords: Sensing; Distributed centralised sensing scheme; Hard-decision; Sensing reporting reduction

\section{Introduction}

The ever increasing demand for higher data rates is unstoppable. A report from CISCO predicts a 39 -fold increase in data traffic in the period 2009 to 2014 [1]. A considerable portion of this data traffic will use wireless infrastructures, increasing the pressure on the efficient management of the available spectrum. The licensed spectrum is largely underutilized [2] as proven by the Federal Communication Commission in [3]. This study shows that the utilization of the licensed spectrum ranges from $15 \%$ to $85 \%$.

Today, it is generally accepted that cognitive radio (CR) [4] is a promising solution to the apparent spectrum scarcity faced by the operators [5]. A CR adapts its operating parameters (i.e. centre frequency, bandwidth, etc.) to avoid interfering with (or be interfered by) other licensed or unlicensed wireless systems in the vicinity. This tuning is based on the radio environment monitoring operation, commonly termed sensing. A comprehensive

\footnotetext{
* Correspondence: carlos.ribeiro@ipleiria.pt

${ }^{2}$ Instituto de Telecomunicações, Instituto Politécnico de Leiria, Alto do Vieiro, Leiria 2411-901, Portugal

Full list of author information is available at the end of the article
}

survey of spectrum sensing techniques can be found in [6]. If a $\mathrm{CR}$ is to autonomously assess the availability of a given channel, it needs to overcome challenging issues posed by the varying wireless channel conditions: hidden node problem, deep fading, shadowing, etc., that will deteriorate CR sensing performance. To alleviate this problem, multiple CRs can cooperate to jointly perform the spectrum sensing, exploiting the multiuser diversity in the sensing process [7-9].

In the cooperative spectrum sensing, each CR usually performs the sensing individually and then reports the local observations to a common fusion unit (FU). This unit gathers the information from all sensors involved in the sensing process, generates the decision on the presence of other users in the channel of interest and broadcasts the decision to the CRs. If a large number of CRs participate in the sensing process, it is expectable that even though some will suffer from the localized sensing limitations (hidden node, deep fading, shadowing and sensing performance), others will output correct reports and the overall decision will be more reliable. A survey on cooperative sensing can be found in [10].

\section{实}


To transmit the local sensing reports to the FU, a bandwidth-limited common reporting channel is generally assumed, and the overhead needed for the CRs to send the reports is a critical issue in cooperative sensing. Although the information by itself may be a simple binary indication, it always requires the setup of the channel, which requires resources. Moreover, the transmissions from the sensors require energy. It is therefore highly convenient for energy reduction and overhead minimization purposes to devise cooperative sensing schemes that minimize the number of transmissions from the localized sensors to the FU.

The reduction of the reporting overhead in cooperative sensing has been addressed in the literature and can be divided into three categories: the CR network throughput optimization schemes [11-13], the user selection schemes [14-17] and the censorship algorithms [18-22].

The CR network throughput optimization schemes aim at maximizing the CR network throughput subject to a given detection probability. The work in [11] optimizes the number of sensors and sensing time that maximizes the network throughput when using energy detection at the sensors and the hard fusion OR decision rule at the FU. An extension for the $k$-out-of- $K$ decision rule at the FU can be found in [12]. An additional energy constraint per $C R$ is added to the problem in [13] to derive the optimal number of sensors, reporting time and probability of false alarm (PFA) that maximizes the throughput.

The user selection schemes use different criteria to select the sensors that will participate in the cooperative sensing, limiting the dimension of the group and lowering the reporting overhead. The confidence level that each sensor builds on its own decision (when comparing with the decisions of remaining sensors) is used in [14] to limit the set of sensors that report back to the FU. The distance to the primary user (PU) is the criteria adopted in [15] for choosing the $k$-out-of- $K$ CR users that will participate in cooperative sensing when the positions of CR users and PU are known. The degree of knowledge of the positions of the CR users is the criteria used in the three selection algorithms proposed in [16] to address the shadow correlation problem in cellular systems. A trade-off between the sensing reliability and the reporting overhead is used in [17] to induce the sensors with the best detection performance to join the cooperative sensing.

The censoring algorithms aim at limiting the number of reports sent by each sensor involved in the cooperative sensing by refraining the CR users from transmitting uninformative data. The local decisions made at each sensor are sent to the FU only if they are considered informative. The most common procedure defines two thresholds as shown in Equation 1. Information is sent only if:

$$
\begin{aligned}
& U>L_{u}(\text { and } 1 \text { is sent }) \\
& \text { or } \\
& U<L_{l}(\text { and } 0 \text { is sent })
\end{aligned}
$$

If the statistic $U$ belongs to the uncertainty interval $L_{l} L_{u}$ then no reporting is performed because the binary information corresponding to the local decision is not reliable enough to justify the usage of transmitted power and radio resources. Although one can significantly reduce the overhead with such a scheme, optimization requires a careful setting of the thresholds $L_{u}$ and $L_{l}$, which are dependent of the probabilities of having a primary signal or not. If such $a$ priori probabilities are not known or cannot be guessed, then a random choice of the thresholds does not guarantee a good receiver operating characteristic (ROC). This can be improved with learning procedures, but this again takes time to stabilize.

To tackle the issue of reducing the sensing report overhead, a new cooperative scheme is proposed that essentially relies on basic information theory principles which say that silence periods may also convey information. The new cooperative sensing scheme reduces the average number of transmissions by $50 \%$ without any loss of performance and could be used in conjunction with the other two groups of distributed sensing algorithms to further reduce the signalling overhead.

The remainder of the paper is organized as follows. The next section introduces the proposed selective reporting algorithm and analyzes its performance under fading channels. Numerical and experimental results are presented and discussed in Section 3. Finally, the main conclusions of this work are drawn in Section 4.

\section{Selective reporting algorithm}

The selective reporting cooperative sensing algorithm explores the information present in silence periods to reduce the sensing report signalling overhead and associated energy and resource usage. The section starts with a concise background review, introduces the proposed cooperative sensing scheme with silence periods and analyzes the distribution of the number of transmitted messages. An information theory interpretation of the new scheme follows, ending with the investigation of the behaviour of the algorithm subject to fading channels.

\subsection{Background review}

Considering a node $k$ out of the $K$ cognitive radios in the scenario of Figure 1, the mathematical model for binary hypothesis testing problem is the following: 


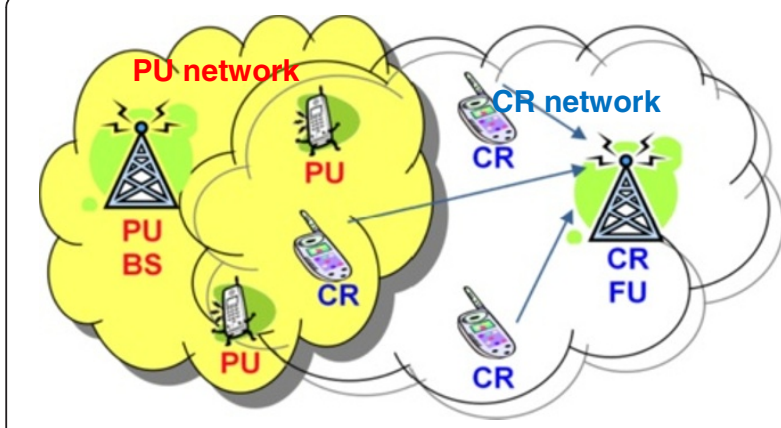

Figure 1 System and concept under analysis.

Primary user absent $\quad H_{0}: \quad y[n]=w[n]$ Primary user present $H_{1}: \quad y[n]=a x[n]+w[n]$

where the following notation is used:

$x[n]$ : transmitted signal, usually modelled as zero-mean additive white Gaussian noise (AWGN) with variance $\sigma_{x}^{2}$ $w[n]$ : zero-mean AWGN noise with variance $\sigma_{w}^{2}$

$y[n]$ : received signal

a: complex coefficient that accounts for channel fading $n=0,1, \cdots,(N-1)$, were $N$ is the number of samples in the observation window of the received signal.

Considering an energy detector, the decision statistic is formed summing up the energy of the received samples during the observation interval, i.e. considering that $N$ samples are collected. The decision statistic $U$ is given by:

$$
U=\frac{1}{N} \sum_{n=0}^{N-1}|y[n]|^{2}
$$

and using this statistic, the classical decision rule is simply:

$$
\left\{\begin{array}{l}
\text { Decide } H_{0} \text { if } U<L \\
\text { Decide } H_{1} \text { if } U \geq L
\end{array}\right.
$$

where $L$ is the decision threshold, chosen as to achieve a given operating point on the sensor's ROC curve.

Let us now extend the observation for the entire distributed sensing scheme of Figure 1. Assuming that the localized sensing variables are sent to the FU, the dominant fusion model has its origins in the distributed detection theory: the parallel fusion technique [23] combines the observations to improve the detection performance. The adoption of this fusion model, and its variants, for cooperative sensing can be found in $[9,24]$.

The combining at the FU can be divided into two major categories depending on the result reported by the sensors. If the sensors report the full samples (or its quantized versions), the FU performs a soft combining, achieving the best performance of both categories, at the cost of a higher signalling load. The optimal soft combining algorithm was proposed by [25], where a Neyman-Pearson criterion was used for the weighted combination of the local samples. If the sensors report the binary local decision, the FU performs a simple hard combining. The most commonly used fusion decision rules are the AND, OR and majority decision. These rules can be generalized in the $k$-out-of- $K$ rule [23] (for $k=1, K,\ulcorner K / 2\rceil$ the rule becomes, respectively, the OR, AND and majority decision rules). The ideal $k$ value depends on the type of channel affecting the transmission, the detection threshold and the total number of sensors [26]. For a large threshold, the OR rule tends to give the best performance, and due to its simplicity, it is commonly used for scenarios where, subject to a given PFA, the goal is to reduce the probability of missed detection (PMD). At each sensor, from Equation 4:

$$
\left\{\begin{array}{l}
\text { Decide } H_{0} \text { if } U<L \text { and send } 0 \\
\text { Decide } H_{1} \text { if } U \geq L \text { and send } 1
\end{array}\right.
$$

The final decision is made at the FU by employing the OR rule: if at least one sensor reports 1 , decides $H_{1}$, otherwise $H_{0}$. With such a rule, and assuming $K$ identical sensors, the global PFA, $q_{D}$, is given by:

$$
q_{D}=1-(1-q)^{K}
$$

where $q$ is the localized (at each sensor) PFA.

Similarly, the PMD, $v_{D}$, is simply expressed as a function of the localized PMDs:

$$
v_{D}=\prod_{i=1}^{K} v_{i}
$$

where $v_{i}$ is the localized PMD for sensor $i(i=1,2, \ldots, K)$.

Equation 6 can be extended, in the case of nonidentical sensors, to:

$$
q_{D}=1-\prod_{i=1}^{K}\left(1-q_{i}\right)
$$

where $q_{i}$ is the localized PFA for sensor $i(i=1,2, \ldots, K)$.

The localized probabilities, considering the energy detector, are amenable to analytical formulation under certain conditions. Assuming that the number of samples $N$ is high so that we can invoke the central limit 
theorem, and resorting to the Gaussian hypothesis, it can be expressed as [27]:

$$
\left\{\begin{array}{l}
q_{i}=Q\left(\frac{K-\sigma_{w(i)}^{2}}{\sigma_{w(i)}^{2} \sqrt{2}} \sqrt{N}\right) \\
v_{i}=Q\left(\frac{K-\left(\sigma_{w(i)}^{2}+\left|a_{i}\right|^{2} \sigma_{x}^{2}\right)}{\sqrt{2\left(\sigma_{w(i)}^{2}+\left|a_{i}\right|^{2} \sigma_{x}^{2}\right)^{2}}} \sqrt{N}\right)
\end{array}\right.
$$

where $a_{i}$ is the complex coefficient that accounts for the channel fading of sensor $i$; $\sigma_{w(i)}{ }^{2}$ is the individual sensor noise variance, and $Q($.$) is the complementary error$ function $\frac{1}{\sqrt{2 \pi}} \int_{x}^{\infty} e^{-x^{2} / 2} d x$.

\subsection{Cooperative sensing with silence periods}

The basis of the proposal relies on the use of two types of sensors that report if different events occur at each one, i.e.:

- Type 1: report only if decision is $H_{1}$. Do not report if decision is $H_{0}$.

- Type 2: report only if decision is $H_{0}$. Do not report if decision is $H_{1}$.

The operating mode of the type 1 sensor is shown in Figure 2 (left). In this figure, the columns represent the hypothesis that is really occurring, and the lines represent the decision made by the sensor. The cells contain the type of message that is sent along with the joint probability of $\left(\hat{H}_{i} ; H_{j}\right) i, j \in\{0 ; 1\}$. The equivalent figure for a type 2 sensor is shown in Figure 2 (right). In this figure, $E$ indicates that no message is sent (from the word erasure) and $M$ that a message is sent. The type of message $(M)$ is rather irrelevant.

The type of node is decided on the initialization procedure, and the FU must keep an up-to-date list with the sensors integrating each group. When a new sensing report arrives, the only thing that counts is the identification of the transmitting node. In fact, upon receiving a message, the FU knows that if it is coming from a type 1 sensor, it means that this node has detected a primary signal, whereas if it is coming from a type 2 sensor, it means that no primary signal was locally detected.

Table 1 illustrates the operation of the scheme considering that there are only two sensors, one type 1 and one type 2 .

Let us consider the number of messages that are transmitted assuming that $P$ type 1 sensors and $P-K$ type 2 sensors are deployed. As a starting assumption, it is considered that localized PMD and PFA are equal for all sensors.

1. Hypothesis $H_{O}$ Let us assume hypothesis $H_{O}$ where no primary signal is present. Conditioned to this hypothesis, a type 1 sensor transmits if it makes an erroneous detection, and thus, the average number of messages $\left(T_{1}\right)$ coming from type 1 sensors is given by:

$$
E\left(T_{1} \mid H_{0}\right)=P q
$$

while type 2 sensors transmit when they make the correct decision, and then, the average number of messages $\left(T_{2}\right)$ coming from type 2 sensors is given by:

$$
E\left(T_{2} \mid H_{0}\right)=(K-P)(1-q) \text {. }
$$

Therefore the average number of transmitted messages is given by:

$$
\begin{aligned}
E\left(T_{1}+T_{2} \mid H_{0}\right) & =P q+(K-P)(1-q) \\
& =P(2 q-1)+K(1-q)
\end{aligned}
$$

2. Hypothesis $H_{1}$ Under hypothesis $H_{1}$, type 1 sensors transmit if they make the correct detection, while type 2 sensors transmit if they miss the detection. Thus, the average number of messages coming from type 1

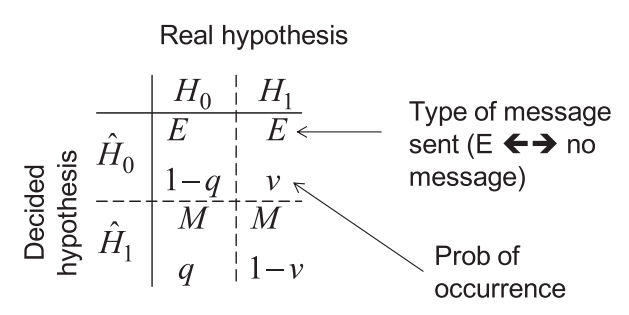

Type 1 sensor

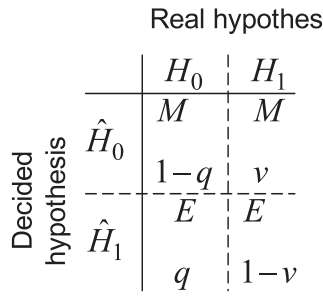

Type 2 sensor

Figure 2 Operation of type 1 (left) sensor and type 2 (right) sensor. 
Table 1 Illustration of the selective reporting scheme with two sensors of different types

\begin{tabular}{|c|c|c|c|c|c|c|}
\hline \multicolumn{3}{|c|}{ Type 1 sensor } & \multicolumn{3}{|c|}{ Type 2 sensor } & \multirow{2}{*}{$\begin{array}{c}\text { FU } \\
\text { decision } \\
\text { (OR rule) }\end{array}$} \\
\hline Local decision & Message sent to FU & Interpretation at FU & Local decision & Message sent to FU & Interpretation at FU & \\
\hline 0 & $E$ & 0 & 0 & $M$ & 0 & 0 \\
\hline 0 & E & 0 & 1 & E & 1 & 1 \\
\hline 1 & M & 1 & 0 & M & 0 & 1 \\
\hline 1 & $M$ & 1 & 1 & E & 1 & 1 \\
\hline
\end{tabular}

and type 2 sensors is given by:

$$
\left\{\begin{array}{l}
E\left(T_{1} \mid H_{1}\right)=P(1-v) \\
E\left(T_{2} \mid H_{1}\right)=(K-P) v
\end{array}\right.
$$

and, therefore, the average number of total transmissions is given by:

$$
E\left(T_{1}+T_{2} \mid H_{1}\right)=P(1-2 v)+K v .
$$

\subsection{Analysis of the distribution of transmitted messages} Let us now consider the distribution of the number of transmitted messages.

1. Hypothesis $H_{0}$

Under the assumptions of identical sensors, the distribution of the number of transmitted messages (see Appendix), conditioned to $H_{0}$, is given by:

$f_{0}(m)=(1-q)^{P} q^{K-P}\left(\frac{1-q}{q}\right)^{m} \sum_{l=0}^{m}\left(\begin{array}{l}P \\ l\end{array}\right)\left(\begin{array}{c}K-P \\ m-l\end{array}\right)\left(\frac{q}{1-q}\right)^{2 l}$

from which the average and variance can be computed:

$$
\left\{\begin{array}{l}
E\left[T \mid H_{0}\right]=P(2 q-1)+K(1-q) \\
\operatorname{Var}\left[T \mid H_{0}\right]=K q(1-q)
\end{array}\right.
$$

2. Hypothesis $H_{1}$

Under the assumption of identical sensors, the distribution of the number of transmitted messages (see Appendix), and respective average and variance conditioned to $H_{1}$, is given by:

$$
\left\{\begin{array}{l}
f_{1}(m)=(1-v)^{K-P} v^{P}\left(\frac{v}{1-v}\right)^{m} \sum_{l=0}^{m}\left(\begin{array}{c}
K-P \\
l
\end{array}\right)\left(\begin{array}{c}
P \\
m-l
\end{array}\right)\left(\frac{1-v}{v}\right)^{2 l} \\
E\left[T \mid H_{1}\right]=P(1-2 v)+K v \\
\operatorname{Var}\left[T \mid H_{1}\right]=K v(1-v)
\end{array}\right.
$$

Now, let us consider what would be the best choice of $P$. Assuming that we have no prior information on the presence or absence of the primary signal, we would like to choose $P$ that minimizes the average value of required transmissions, whatever the value of the hypothesis,

$$
\min _{P}\left\{\max \left\{E\left[T \mid H_{0} ; P\right] ; E\left[T \mid H_{1} ; P\right]\right\}\right\}
$$

The problem is illustrated graphically in Figure 3. The thick segments represent the maximum value of the average required transmissions for both hypotheses. The optimum values is achieved when

$$
\begin{aligned}
& E\left[T \mid H_{0}\right]=E\left[T \mid H_{1}\right] \Leftrightarrow \\
& P(2 q-1)+K(1-q)=P(1-2 v)+K v \Leftrightarrow \\
& (2 P-K)(q+v-1)=0
\end{aligned}
$$

For the values of PFA, $q$ and PMD, $v$, of interest, the only solution to Equation 19 is $P=K / 2$. This result will be used throughout the remaining of the paper.

Let us update the solution of Equation 19 in the most relevant equations. Under hypothesis $H_{0}$, Equation 12 can be written as:

$$
E\left(T_{1}+T_{2} \mid H_{0}\right)=\frac{K}{2}(2 q-1)+K(1-q)=\frac{K}{2} .
$$

The result in Equation 20 proves that, irrespective of the localized PFA, the average number of transmissions is $K / 2$, i.e. in average, only half of the sensors transmit.

The distribution of the number of transmitted messages, conditioned to $H_{O}$, is given by:

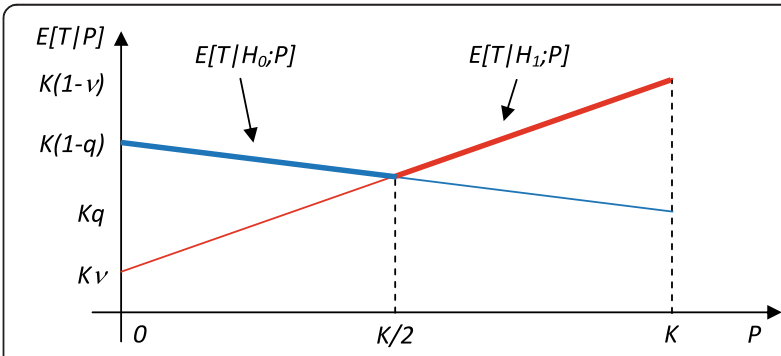

Figure 3 Illustration of the problem of minimizing the average value of required transmissions. 
$f_{0}(m)=(1-q)^{K / 2} q^{K / 2}\left(\frac{1-q}{q}\right)^{m} \sum_{l=0}^{m}\left(\begin{array}{c}K / 2 \\ 1\end{array}\right)\left(\begin{array}{c}K / 2 \\ m-l\end{array}\right)\left(\frac{q}{1-q}\right)^{2 l}$

The variance is given by Equation 16 .

Under hypothesis $H_{1}$, Equation 14 can be written as:

$$
E\left(T_{1}+T_{2} \mid H_{1}\right)=\frac{K}{2}(1-2 v)+K v=\frac{K}{2}
$$

Irrespective of the localized PMDs, the average number of transmissions is $K / 2$, i.e. in average, only half of the sensors transmit.

From Equations 20 and 22, one concludes that, irrespective of the hypothesis, the average number of transmissions from the sensors to the $\mathrm{FU}$ is $K / 2$, which means that, when there is no fading, the average number of messages when using a total of $K$ sensors with half being of type 1 and half of type 2 is equal to $K / 2$.

The distribution of the number of transmitted messages, conditioned to $H_{1}$, is given by:

$$
\begin{aligned}
f_{1}(m)= & (1-v)_{v}^{K / 2}\left(\frac{v}{1-v}\right)^{m} \\
& \sum_{l=0}^{m}\left(\begin{array}{c}
K / 2 \\
l
\end{array}\right)\left(\begin{array}{c}
K / 2 \\
m-l
\end{array}\right)\left(\frac{1-v}{v}\right)^{2 l}
\end{aligned}
$$

The variance is given by Equation 17 .

As the PFA and PMD coincide with the ones of $K$ sensors with classical transmission/detection employing an $\mathrm{OR}$ rule at the $\mathrm{FU}$, it can be concluded that with the proposed scheme, the number of transmissions can be reduced by a factor of 2 with no penalty in the ROC.

For typical designs, the number of transmissions is much more concentrated around the mean for hypothesis 0 than for hypothesis 1 . Let us just point out some numbers for a scenario with ten sensors (five of each type). If the global PFA is designed to be $q_{D}=0.2$, using Equation 6, this requires a localized PFA at each sensor $q_{i}=0.022$, and therefore, $\sigma_{H_{0}}=\sqrt{\operatorname{Var}[T \mid] H_{0}=\sqrt{10 \times 0.022 \times(1-0.022)}=0.46}$, whereas designing for a global PMD $v_{D}=10^{-5}$, using Equation 7, the required localized PMD is $v_{i}=0.316$, resulting in

$\sigma_{H_{1}}=\sqrt{\operatorname{Var}\left[T \mid H_{1}\right]=\sqrt{10 \times 0.316 \times(1-0.316)}=1.47}$.

It may be surprising that with the selective reporting scheme one gets, with fewer transmissions, the same ROC as in the case of a conventional distributed detector, where the results of the local detection are always sent. This may be explained using basic concepts of information theory which states that no transmission (silent periods) may carry information. Let us consider two sensors (one type 1 and one type 2) as shown in Figure 4, where $E$ stands for no transmission.

It is easy to verify that the mutual information between A and B is the same if one replaces, in the output alphabet of type 1 sensors, $E$ by 0 , and, for the type 2 sensors, $E$ by 1 . The mutual information can be increased if we allow the use of three symbols, leaving the promise that using a double threshold can lead to improved performance.

\subsection{Algorithm behaviour with fading channels}

Let us now consider the case of fading between the primary system and the sensors. It is clear that when the primary signal is absent, nothing changes. Assuming that the sensors are identical, Equations 20 and 21 can still be used, i.e. it can be expected that the average number of transmissions is still equal to half the number of sensors.

However, the analysis under hypothesis $H_{1}$ is different. As the signal from the primary system arrives at the sensors with different powers, the PMD is different for the various sensors.

Let us now consider hypothesis $H_{1}$, assuming $K$ sensors labelled $0,1, \ldots, K / 2-1$ for sensors of type 1 and $K / 2$, $K / 2+1, \ldots, K-1$ for sensors of type 2 . The PMD $i, v_{i}$, is given (under the asymptotic Gaussian approximation) by Equation 9. Sensors of type 1 transmit therefore with probability $\left(1-v_{i}\right)$ and sensors of type 2 with probability $v_{i}$. Although an analytic expression for the distribution of the number of transmissions is not amenable, the first and second order moments are quite easy to derive since it is a sum of Bernoulli variables that are independent. The additivity of the mean and variance can be invoked. The average number of transmissions for sensor $i$ is given by:

$$
E\left[T_{i} \mid H_{1}\right]=\left\{\begin{array}{cc}
1-v_{i} & 0 \leq i \leq K / 2-1 \\
v_{i} & K / 2 \leq i \leq K-1
\end{array}\right.
$$

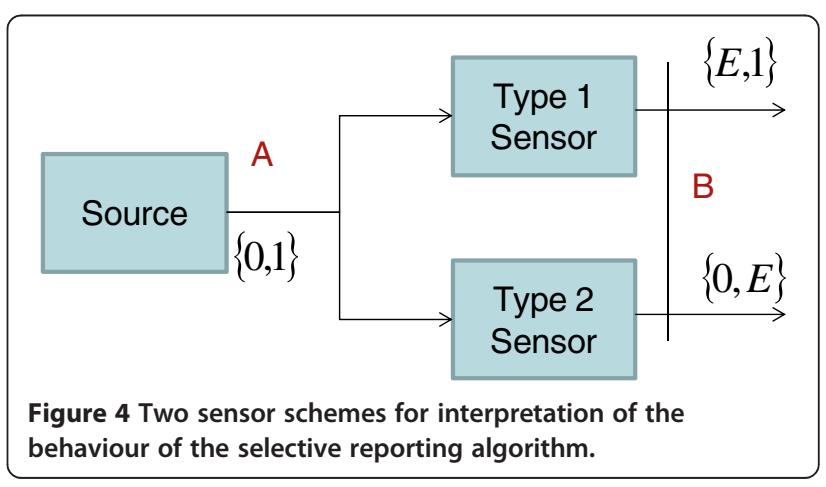




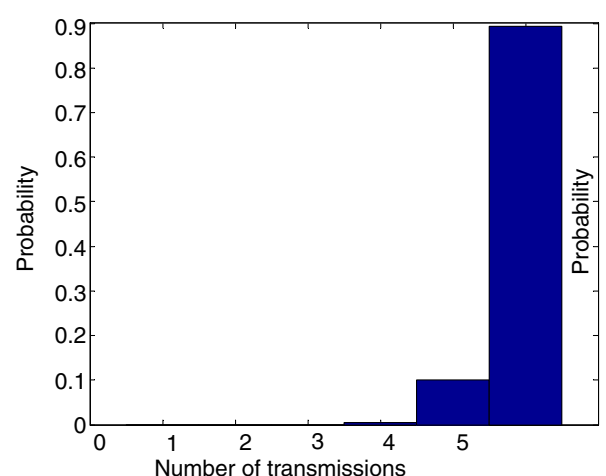

a) Type 2

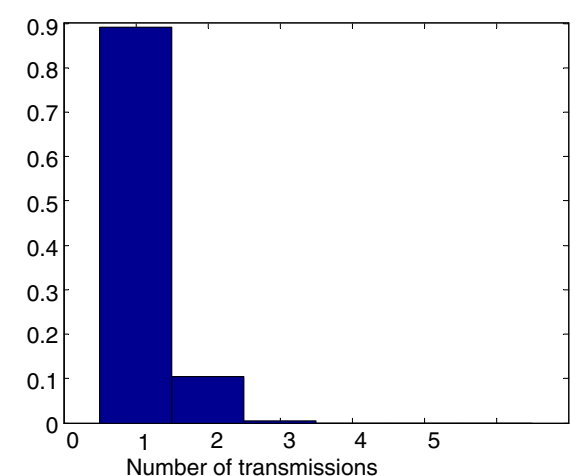

b) Type 1

Figure 5 Distribution of the number of transmissions for (a) type 2 and (b) 1 nodes; hypothesis $H_{0}$.

Therefore,

$$
\begin{aligned}
E\left[T \mid H_{1}\right] & =\sum_{i=0}^{K / 2-1} 1-v_{i}+\sum_{i=K / 2}^{K-1} v_{i} \\
& =\frac{K}{2}+\sum_{i=0}^{K / 2-1} v_{i+K / 2}-v_{i}
\end{aligned}
$$

Over the long term, if the environment is stationary, the average value of transmissions will be $K / 2$, but for the reporting at a specific instant, this will depend on the specific distributions of the fading from the primary source to the sensors.

The variance of a Bernoulli variable with parameter $\alpha$ is $\alpha(1-\alpha)$. Therefore, for a sensor, we get a variance $v_{i}\left(1-v_{i}\right)$, either with parameter $v_{i}$ or $\left(1-v_{i}\right)$. As the variables are independent, we get, for the total number of transmissions, the variance:

$$
\operatorname{var}\left[T \mid H_{1}\right]=\sum_{i=0}^{K-1}\left(1-v_{i}\right) v_{i}
$$

By introducing a new level of randomness in the PMDs, the variability of the number of transmissions will clearly increase.

\section{Performance assessment}

This section presents numerical results related to the number of transmissions achieved with the proposed algorithm and experimental results of the implementation of the selective reporting scheme in a distributed sensing test bed [28].

\subsection{Numerical results}

To assess the performance of the proposed algorithm, several simulations were performed with the following parameters:
- Number of sensing nodes: 10

- Type 1 nodes: 5

- Type 2 nodes: 5

- Global PFA designed for $q_{D}=0.1$

- Transmitted signal and noise Gaussian

- Signal to noise ratio at the sensors in the case a primary signal is present

- $0 \mathrm{~dB}$ for the case when no channel fading is considered

- Uniformly distributed between $[-8 ; 10] \mathrm{dB}$ for the case when fading is considered

- Number of samples used in the energy detector: 20

Figures 5 and 6 report the distribution of the number of transmissions for the two different types of nodes for the case where no primary signal is present.

Figure 5 clearly shows the expected asymmetry between the distributions. The type 1 nodes are silent most

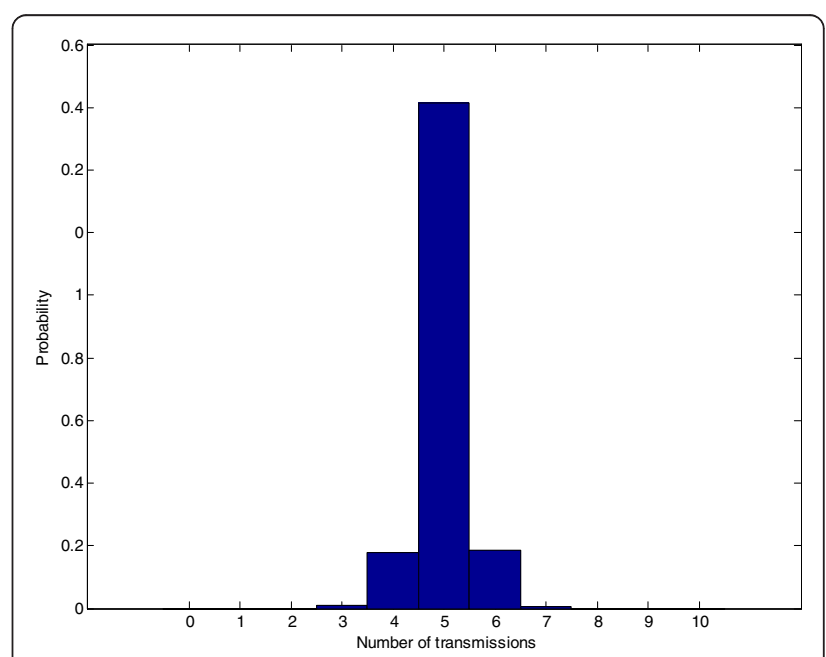

Figure 6 Distribution of the total number of transmissions; hypothesis $H_{0}$. 


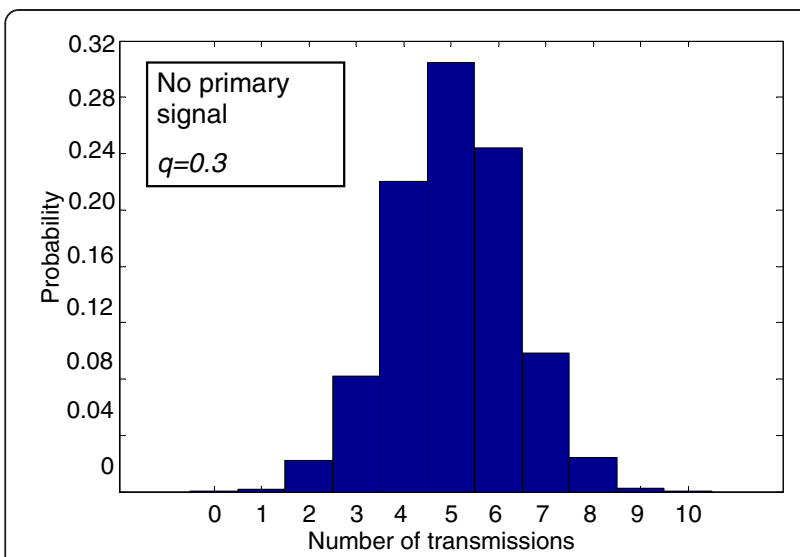

Figure 7 Distribution of the total number of transmissions. PFA designed for 0.3 ; hypothesis $\mathrm{H}_{0}$.

of the time whereas the type 2 nodes are transmitting with a high probability.

In Figure 6, it is easy to identify the symmetry around the average value which is equal to half the number of sensors, i.e. five, with the distribution highly concentrated near this value.

The average value of transmissions in the case reported in Figure 6 is very close to the statistical mean as it can be seen by the high concentration of transmissions around five and the symmetry of the histogram. A wider spread will occur if the threshold is changed to give a larger PFA. However, to notice significant asymmetry, one has to increase the PFA to 0.3 , which is a design value seldomly used in practical systems. The resulting distribution is shown in Figure 7 where a much larger dispersion around the mean and an asymmetry

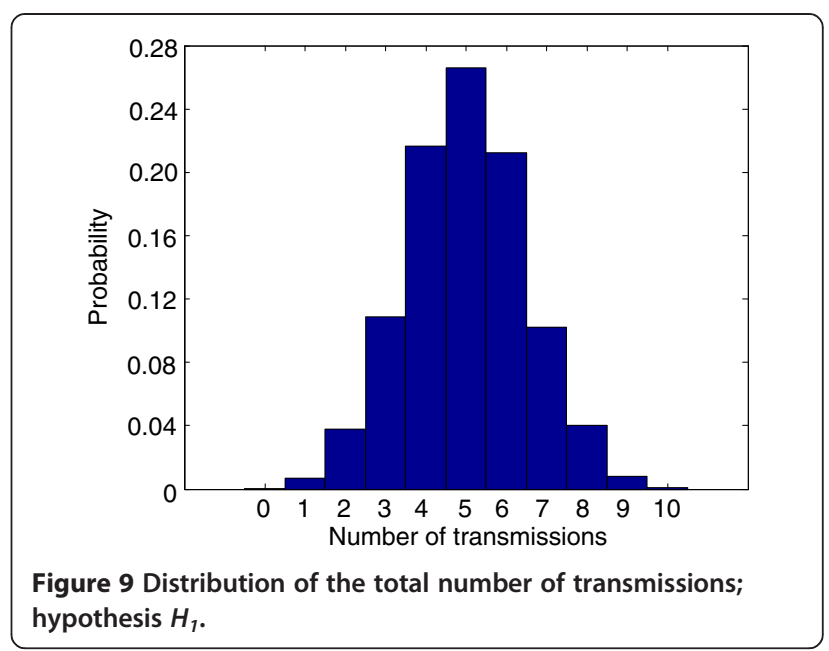

can be seen (in this particular simulation for values above the mean).

Figures 8 and 9 report the distribution of the number of transmissions for the two different types of nodes for the case where the primary signal is present. No fading is assumed, and the path loss is such that the signal-to-noise ratio (SNR) at each node is equal to $0 \mathrm{~dB}$. In these figures, a larger spread in the distributions can be seen, as it was anticipated in the theoretical analysis. However, the mean is very close to half the number of nodes in Figure 9.

In Figure 10, the behaviour in the average number of transmissions is evaluated as a function of the SNR (equal for all nodes). The figure shows that within a 12 $\mathrm{dB}$ fluctuation range, the results are very close to the theoretical statistical mean.

Figures 11 and 12 show the probability distributions in the presence of fading, i.e. the SNRs at each node may be

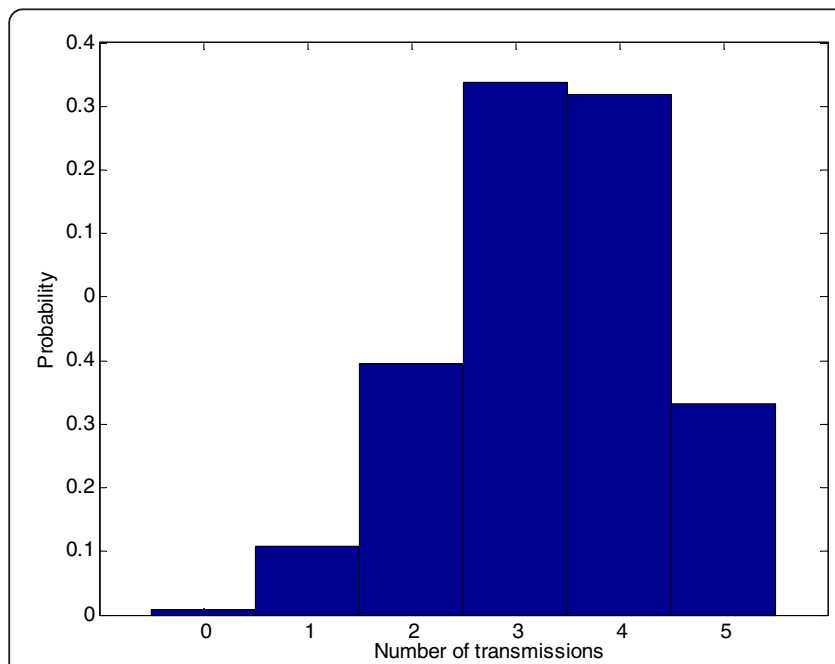

a)

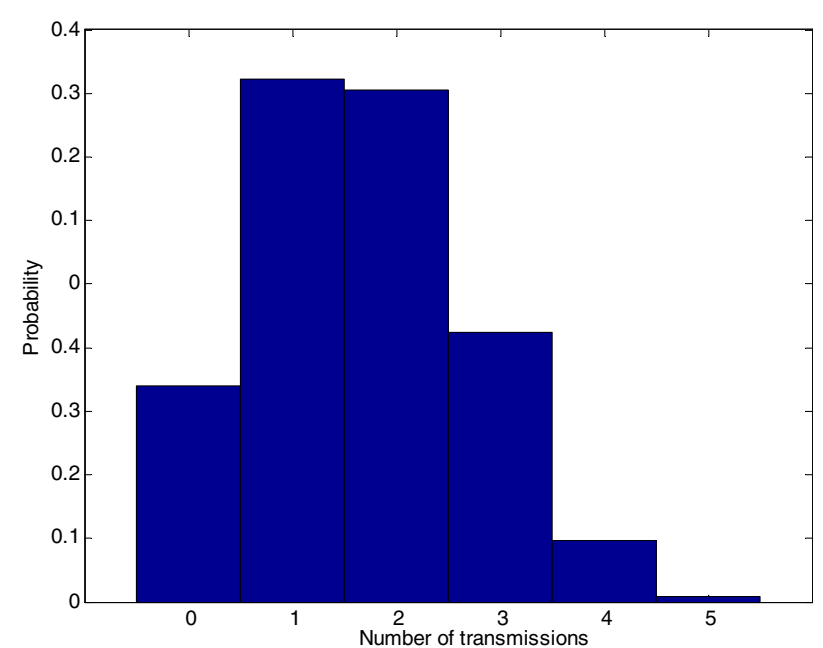

b)

Figure 8 Distribution of the number of transmissions. For the type 2 (a) and type 1 (b) nodes; hypothesis $H_{1}$ and no fading. 


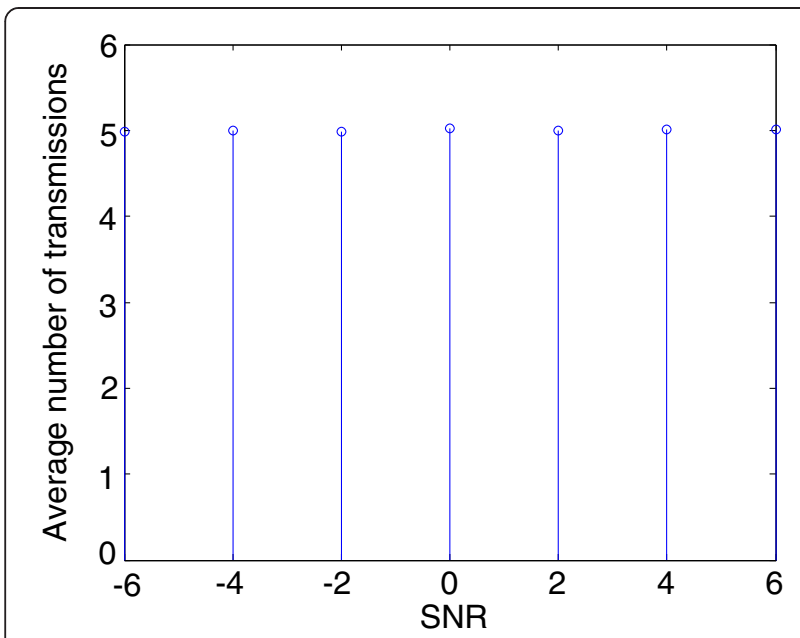

Figure 10 Average number of transmissions as a function of the SNR. Hypothesis $H_{1}$, with no fading.

different. For these figures, the SNR is assumed to vary uniformly in the interval $[-8 ; 10] \mathrm{dB}$. Figure 11 shows a wider spread in the number of transmissions. In both plots ( $a$ and $b$ ), one can notice that at the opposite edge of the point where the probability is expected to be maximum there is an increase in the density. This arises because, for type 1 sensors, there may be nodes with very low SNR that simply cannot detect the primary signal and contribute to an enhancement of the value of 0 . The opposite arises for type 2 nodes. Nevertheless, the distribution of Figure 12 turns out to be very symmetric, and the mean is very close to 5 .

To evaluate the expected value of the number of transmissions, higher deviations in the SNR span were considered. In Figure 13, four cases are presented. The SNRs at the different nodes may vary in the intervals [ $-8 k ; 10 k] \mathrm{dB}$ with $k$ taking the values $1,2,3$ and 4 . The results show that although the SNRs vary, the average

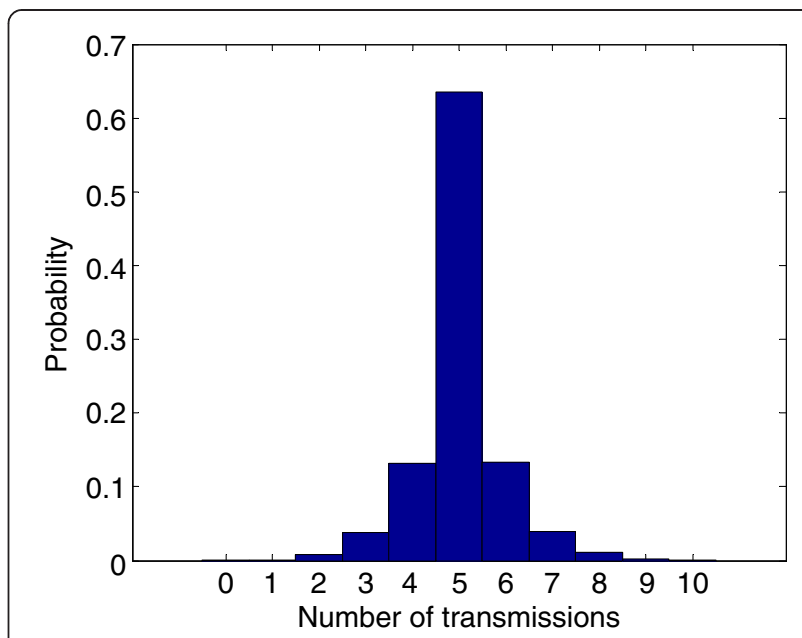

Figure 12 Distribution of the total number of transmissions. Hypothesis $\mathrm{H}_{1}$ and fading leading to SNR uniformly distributed in $[-8 ; 10] \mathrm{dB}$.

number of transmissions is still very close to the theoretical statistical value. In general, one can say that for the parameters of interest (that can be summarized as $q_{D}<0.1$ and $v_{D}<10^{-3}$ ) and for a moderately large number of sensors, the distributions are quite sharp and the number of transmissions are very close to the mean.

\subsection{Experimental results}

The selective reporting scheme was implemented in the distributed sensing test bed depicted in Figure 14 to evaluate the performance of the proposed algorithm in a closeto-real scenario. The test bed was composed of four sensing nodes, a FU and a primary scene emulator (baseband signal generator, channel emulator and RF upconverters). The sensing nodes and the FU were implemented in the open SDR platform GNU Radio [29]. The sensors used Ettus USRP [30] hardware to

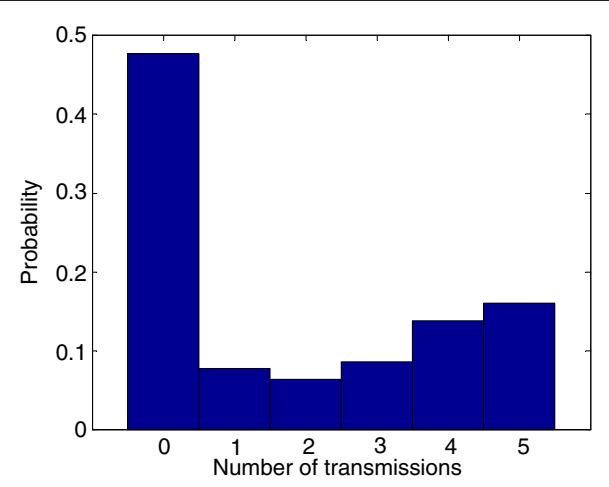

a)

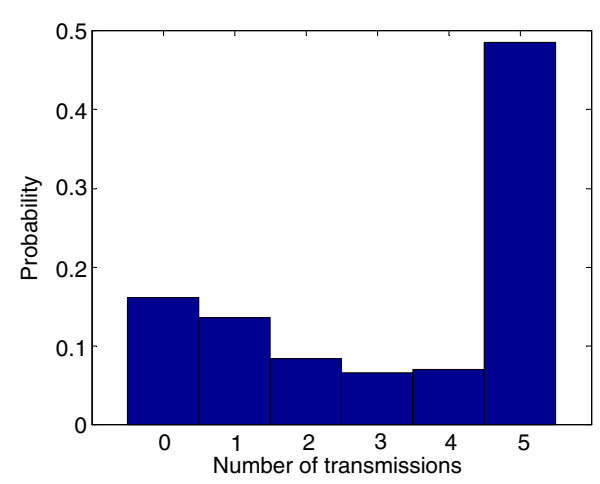

b)

Figure 11 Distribution of the number of transmissions for type 2 (a) and type 1 (b) nodes. Hypothesis $H_{1}$ and fading leading to SNR uniformly distributed in $[-8 ; 10] \mathrm{dB}$. 


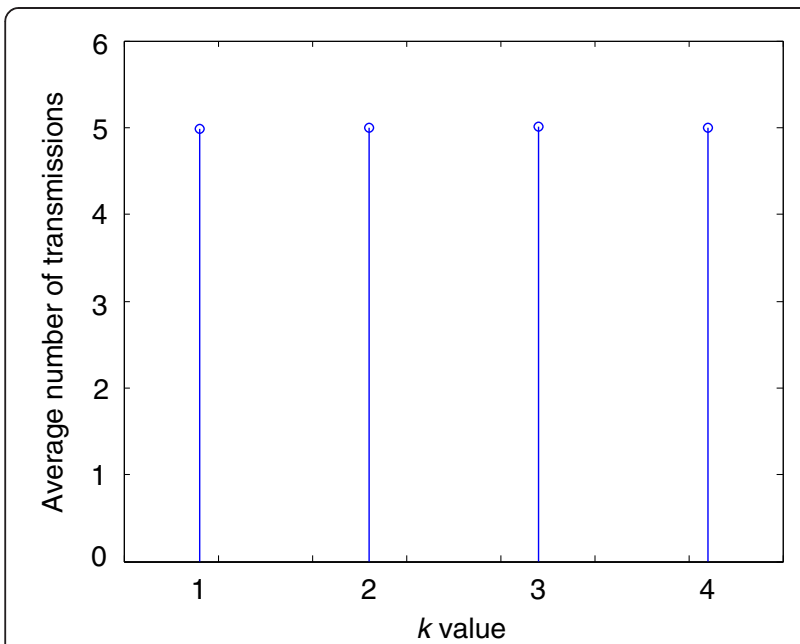

Figure 13 Average number of transmissions as a function of the SNR. Hypothesis $H_{1}$ and fading leading to different spans for the SNR.

implement the physical layer algorithms. The sensors communicate with the FU over standard Fast Ethernet. The primary scene emulation was performed by the Agilent Technologies, Santa Clara, CA, USA [31] equipments in Figure 14. Further details on the test bed can be found in [28]. The main parameters used in the tests were as follows:

- Number of sensing nodes: 4

$\rightarrow$ Type 1 nodes: 2

$\rightarrow$ Type 2 nodes: 2

- Primary signal represented by an $8 \mathrm{MHz}$ bandwidth Digital Video Broadcasting - Terrestrial signal with a $0.8 \mathrm{GHz}$ carrier frequency

- Tests performed with and without independent fading channels; used fading channel model - LTE extended typical urban low Doppler channel [32]

- SNR at the sensors in the case a primary signal is present: $\{0 \mathrm{~dB}, 2 \mathrm{~dB}\}$
- Number of samples used in the energy detector: $\{10$, $20, \ldots, 90\}$

- Local decision thresholds: $\{0 \mathrm{~dB}, 1 \mathrm{~dB}, 2 \mathrm{~dB}\}$

Figures 15, 16, 17 and 18 show the ROC gains [28] of using a distributed sensing scheme with four sensors and an OR decision rule implemented in the FU, when compared to a single sensor. The plots in Figures 15 and 16 show the ROC gains when no fading channel is affecting the received signal and all sensors have similar SNR $(0 \mathrm{~dB}$ and $2 \mathrm{~dB}$, respectively, in Figures 15 and 16). The plots in Figures 17 and 18 depict the ROC gains when independent fading channels affect the signals received by the different sensors. Like in the previous figures, the average SNR of all sensors is similar $(0 \mathrm{~dB}$ and $2 \mathrm{~dB}$, respectively, in Figures 17 and 18). The PMD gains are clearly visible for low values of PFA and decrease as PFA values increase, because the single sensor's probability of detection approaches the unit value.

Figure 19 presents a comparison of the PFA for the four-sensor OR decision rule distributed sensing and four-sensor selective reporting. The plots show that for the tested thresholds, there is no significant difference in the performance between the reference OR-based scheme and the proposed scheme. Figures 20 and 21 plot the PMD differences between the proposed scheme and the reference OR-based, with and without fading channel. Observing both figures, one can easily conclude that the PMD difference is negligible, proving that the proposed method yields the same performance as the reference.

The plots in Figure 22 report the distribution of the number of transmissions (left vertical axis) for the two different types of nodes for different values of PFA (right vertical axis) when no primary signal is present.

In coherence with the theoretical analysis and the numerical results presented in the plots of Figure 5, the asymmetry between the distributions of type 1 and type 2 nodes is clearly visible. As the PFA decreases, two consequences can be observed: type 2 nodes (top plot)

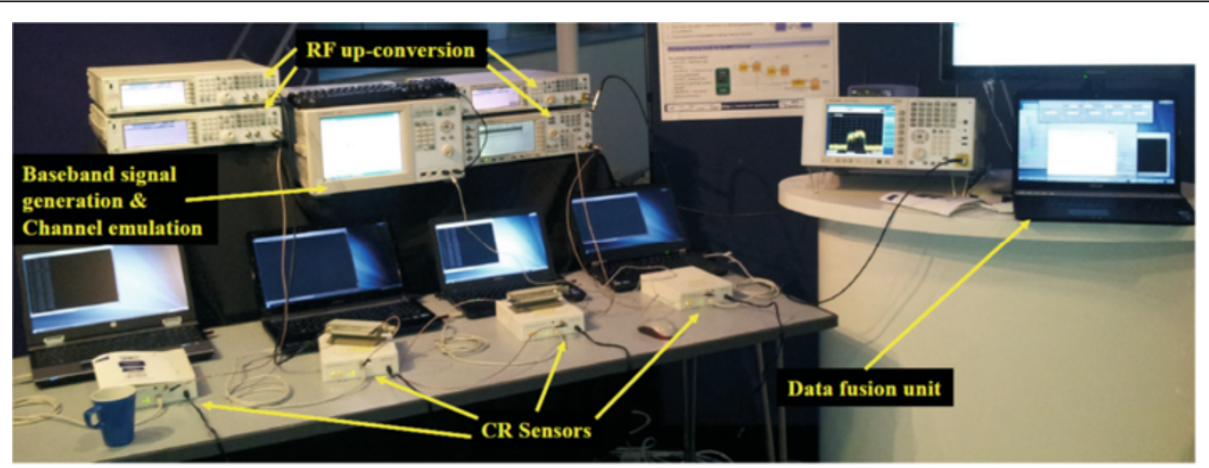

Figure 14 Picture of the test bed implementation. 


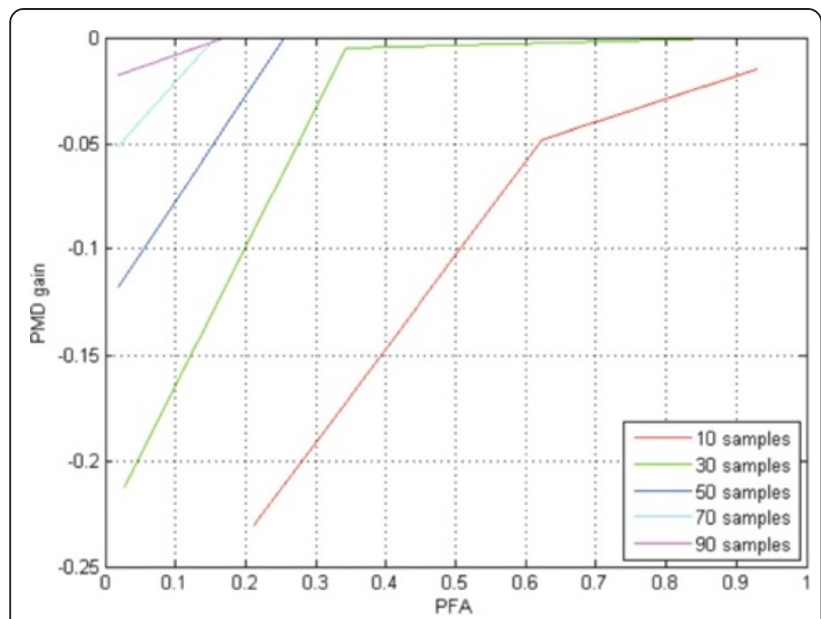

Figure 15 PMD gain of four sensors with FU OR algorithm ( $S N R=0 \mathrm{~dB}$, no channel).

become increasingly silent and type 1 nodes (bottom plot) transmit with a high probability; the distribution of the number of transmissions becomes narrower.

Figure 23 reports the distribution of the total number of transmissions (left vertical axis) for different values of PFA (right vertical axis) when no primary signal is present. As observed previously in Figure 22, as the PFA decreases, the distribution of the number of transmissions becomes narrower. Another consequence that should be pointed is the asymmetry of the distribution. The leftmost set of bars (corresponding to the lowest PFA) presents a distribution shifted to the right, in coherence to the numerical results depicted in Figure 7. As the PFA increases, the distribution widens and the distribution shifts to the left. The rightmost set of bars (corresponding to the largest PFA $>0.5$ ) already shows a left-shifted distribution that, actually, can be correlated with the results presented in Figure 9,

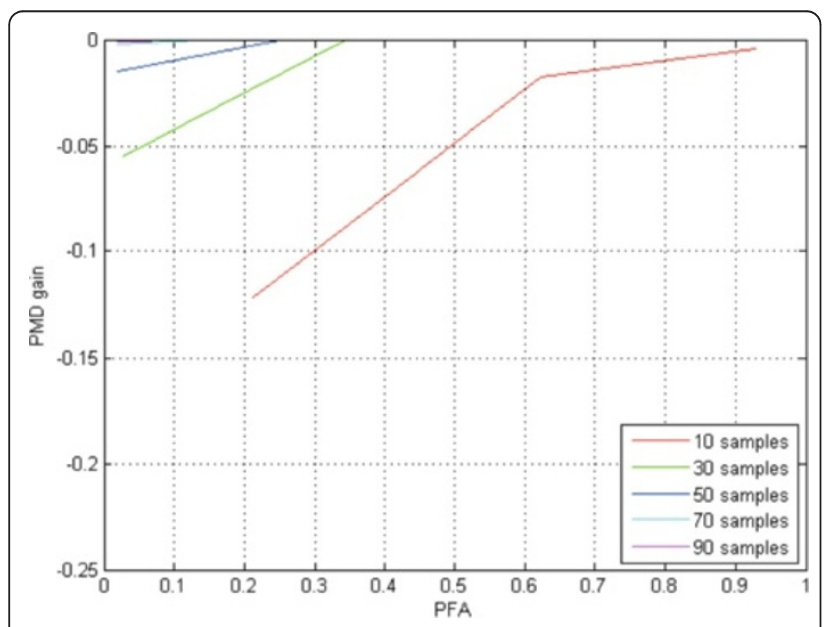

Figure 16 PMD gain of four sensors with FU OR algorithm (SNR $=2 \mathrm{~dB}$, no channel).

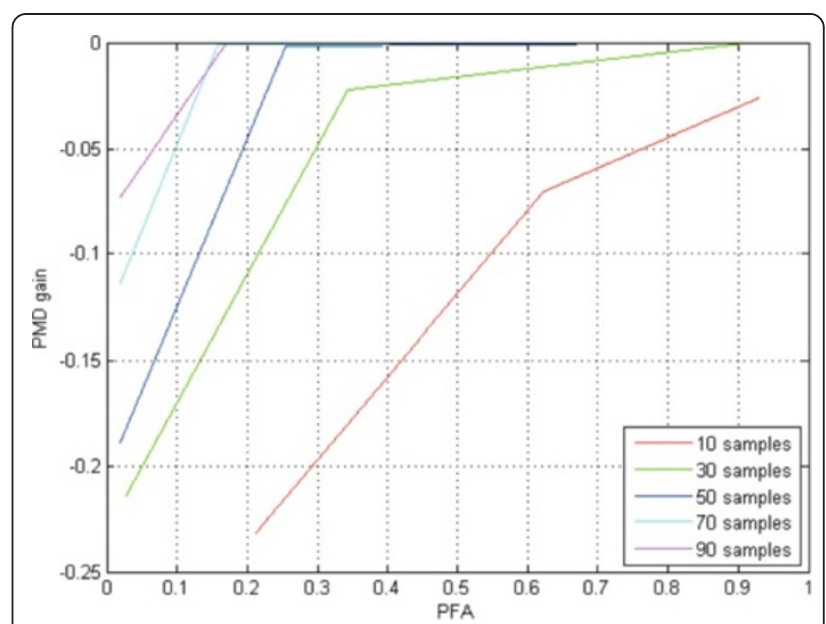

Figure 17 PMD gain of four sensors with FU OR algorithm ( $S N R=0 \mathrm{~dB}$, channel).

associated with the case where the primary signal is present.

\section{Conclusions}

The use of cognitive radios is becoming more and more an unquestionable reality to fight the apparent spectrum scarcity and efficiently explore the available spectrum. Spectrum sensing is a fundamental tool of cognitive radios to acquire the spectrum and decide on the best resources to use. The multiuser diversity provided by cooperative spectrum sensing schemes boosts the reliability of the spectrum acquisition, but the available sensing report channels easily become the bottleneck to the number of participants.

This paper proposes a new cooperative sensing scheme that introduces silence periods in the sensing reporting to lower the reporting bandwidth by $50 \%$.

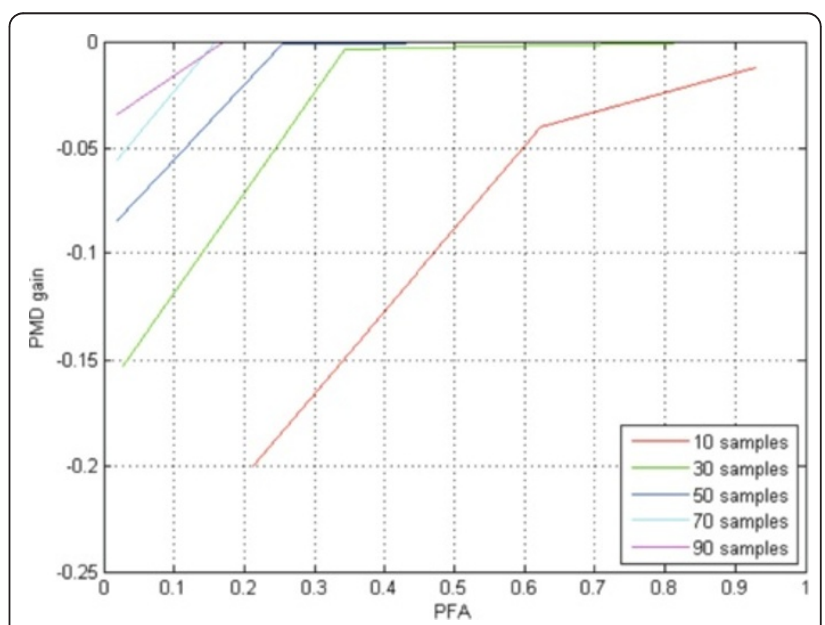

Figure 18 PMD gain of four sensors with FU OR algorithm (SNR $=2 \mathrm{~dB}$, channel) 


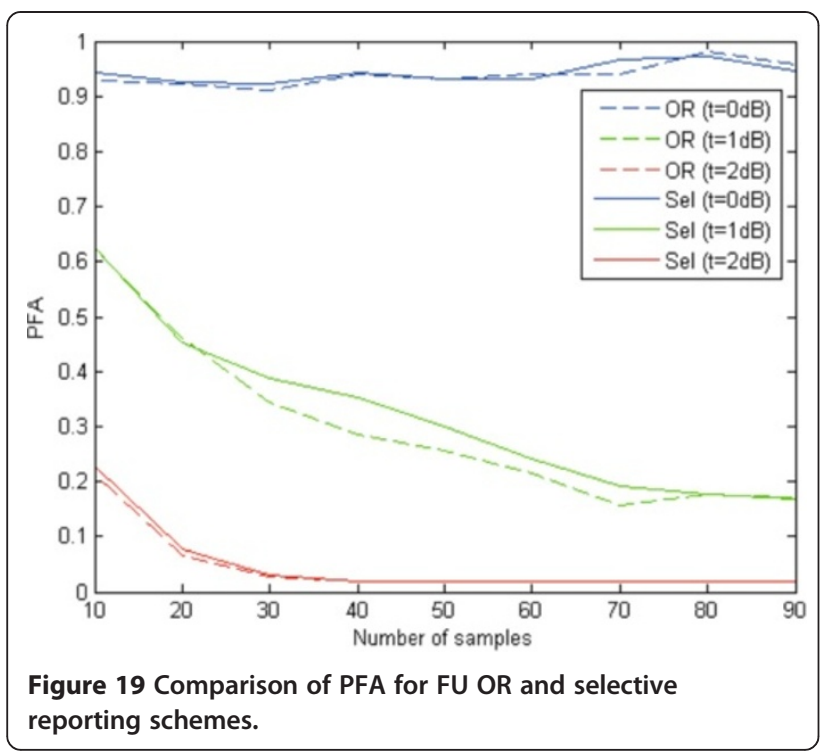

Using the principles of information theory, the algorithm explores the information present in the silence periods to maintain the same performance of standard reporting schemes. The article presents a theoretical analysis of the distribution of transmitted messages, an information theory interpretation of selective reporting sensing scheme and a study on the behaviour of the algorithm when the received signal is subject to fading caused by the wireless channel that links primary transmitter and cognitive radio user. The theoretical analysis is backed up by numerical and experimental results that show that the PFA and PMD are similar to standard reporting schemes, validating the proposed algorithm. Furthermore, the proposed scheme can be used jointly with censorship and user selection algorithms to further

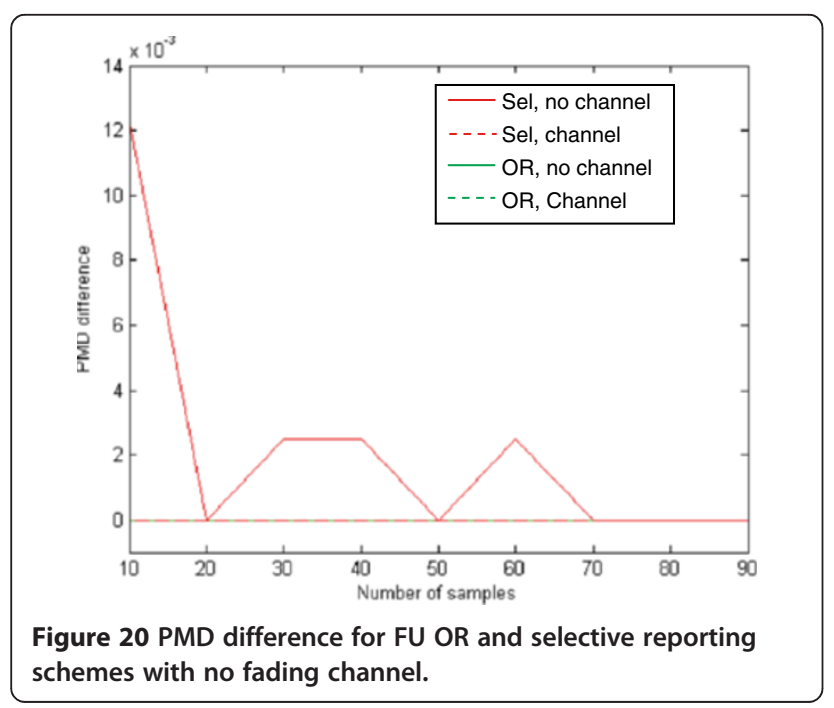

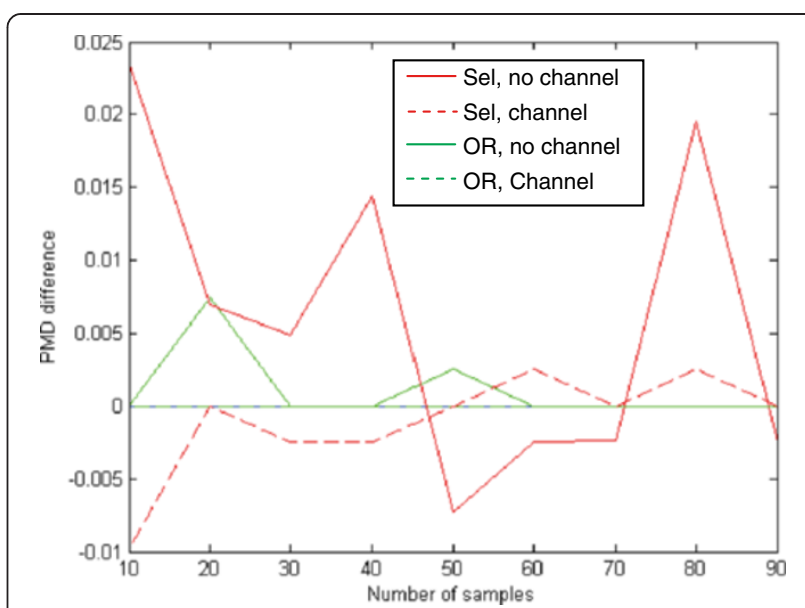

Figure 21 PMD difference for FU OR and selective reporting schemes with fading channel.

reduce the bandwidth demanded of the sensing report channel.

\section{Appendix}

Under the assumptions of identical sensors and hypothesis $H_{0}$, the transmission of type 1 sensors represents $P$ independent and identically distributed (i.i.d.) Bernoulli trials. The variable that accounts for the number of transmitted messages coming from these sensors follows a binomial distribution, $X_{0}^{T 1} \sim B(P, q)$ with a probability distribution function:

$$
f_{0}^{T 1}(l ; P, q)=\left(\begin{array}{c}
P \\
l
\end{array}\right) q^{l}(1-q)^{P-l} .
$$

The transmission of type 2 sensors represents $K-P$ i.i.d. Bernoulli trials. The variable that accounts for the number of transmitted messages coming from these sensors follows a binomial distribution, $X_{0}^{T 2} \sim B(K-P, 1-q)$ with a probability distribution function:

$$
f_{0}^{T 2}(l ; K-P, 1-q)=\left(\begin{array}{c}
K-P \\
l
\end{array}\right)(1-q)^{l} q^{K-P-l} .
$$

Considering that $X_{0}^{T 1}$ and $X_{0}^{T 2}$ are independent, the distribution of the overall number of transmitted messages, conditioned to $H_{0}$, is therefore given by:

$$
\begin{aligned}
f_{0}(m) & =\sum_{l=0}^{m} f_{0}^{T 1}(l ; P, q) f_{0}^{T 2}(m-l ; K-P, 1-q) \\
& =\sum_{l=0}^{m}\left(\begin{array}{c}
P \\
l
\end{array}\right) q^{l}(1-q)^{P-l}\left(\begin{array}{c}
K-P \\
m-l
\end{array}\right)(1-q)^{m-l} q^{K-P-m+l} \\
& =(1-q)^{P} q^{K-P}\left(\frac{1-q}{q}\right)^{m} \sum_{l=0}^{m}\left(\begin{array}{c}
P \\
l
\end{array}\right)\left(\begin{array}{c}
K-P \\
m-l
\end{array}\right)\left(\frac{q}{1-q}\right)^{2 l}
\end{aligned}
$$



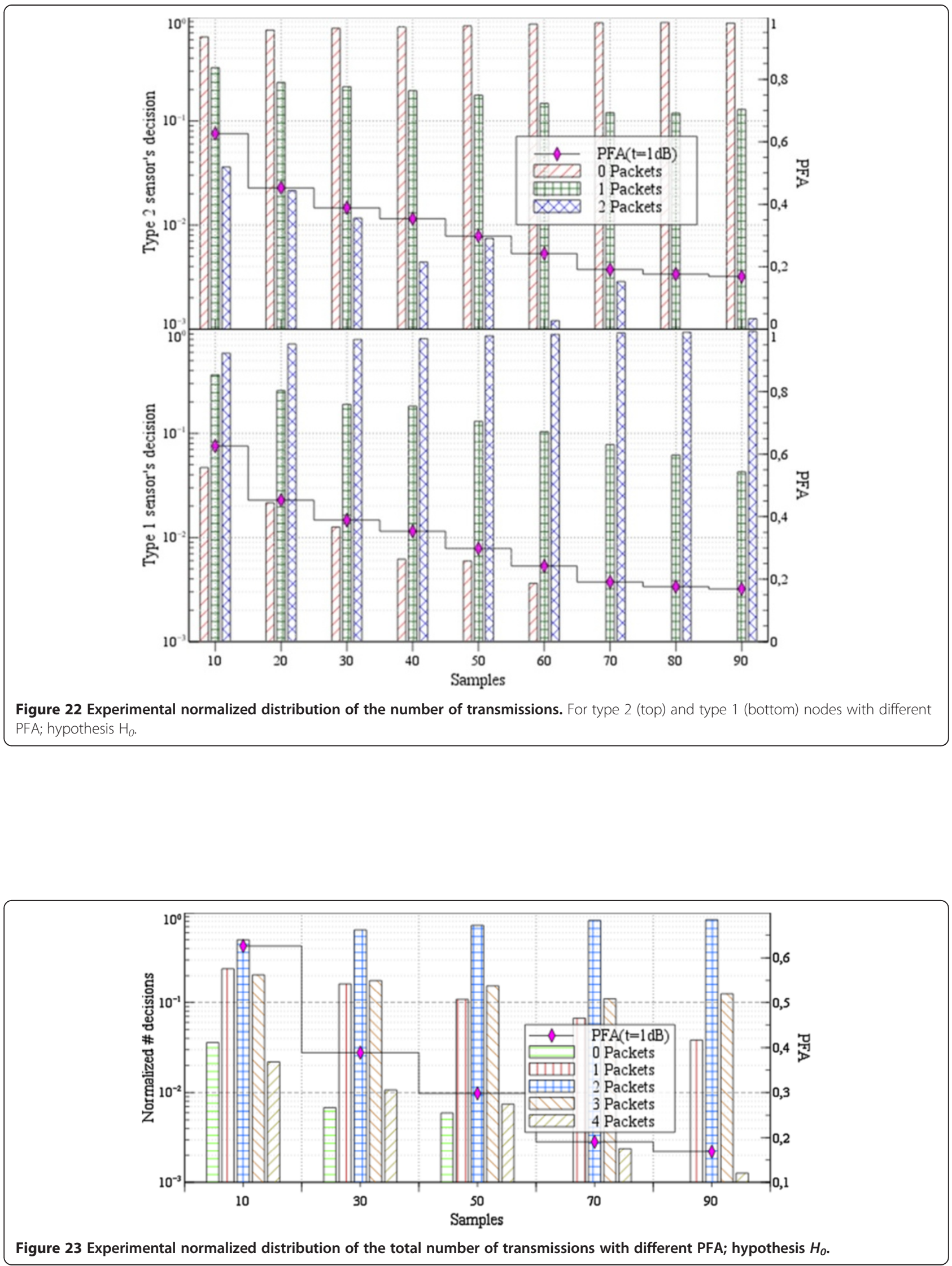
For hypothesis $H_{1}$, the transmission of type 1 sensors represents $P$ i.i.d. Bernoulli trials with probability $(1-v)$, $X_{1}^{T 1} \sim B(P, 1-v)$, with a probability distribution function:

$$
f_{1}^{T 1}(l ; P, 1-v)=\left(\begin{array}{c}
P \\
l
\end{array}\right)(1-v)^{l} v^{P-l}
$$

For type 2 sensors, we have $X_{1}^{T 2} \sim B(K-P, v)$, with a probability distribution function:

$$
f_{1}^{T 2}(l ; K-P, v)=\left(\begin{array}{c}
K-P \\
l
\end{array}\right) v^{l}(1-v)^{K-P-l}
$$

The distribution of the overall number of transmitted messages, conditioned to $H_{1}$, is therefore given by:

$$
\begin{aligned}
f_{1}(m) & =\sum_{l=0}^{m} f_{1}^{T 1}(l ; P, 1-v) f_{1}^{T 2}(m-l ; K-P, v) \\
& =\sum_{l=0}^{m}\left(\begin{array}{c}
P \\
l
\end{array}\right)(1-v)^{l} v^{P-l}\left(\begin{array}{c}
K-P \\
m-l
\end{array}\right) v^{m-l}(1-v)^{K-P-m+l} \\
& =(1-v)^{K-P} v^{P}\left(\frac{v}{1-v}\right)^{m} \sum_{l=0}^{m}\left(\begin{array}{c}
P \\
l
\end{array}\right)\left(\begin{array}{c}
K-P \\
m-l
\end{array}\right)\left(\frac{1-v}{v}\right)^{2 l}
\end{aligned}
$$

\section{Competing interests}

The authors declare that they have no competing interests.

\section{Acknowledgements}

The research leading to these results was derived from the European Community Seventh Framework Programme (FP7) under Grant Agreement number 248454 (QOSMOS)

\section{Author details}

${ }^{1}$ Instituto de Telecomunicações, Universidade de Aveiro, Aveiro 3810-193, Portugal. ${ }^{2}$ Instituto de Telecomunicações, Instituto Politécnico de Leiria, Alto do Vieiro, Leiria 2411-901, Portugal.

\section{Received: 1 April 2013 Accepted: 4 July 2013}

Published: 15 July 2013

\section{References}

1. M Reardon, Cisco predicts wireless data explosion. http://news.cnet.com/ 8301-30686_3-10449758-266.html. Accessed 1 April 2013

2. G Staple, K Werbach, The end of spectrum scarcity. IEEE Spectrum 41(3), 48-52 (2004)

3. Federal Communications Commission, Spectrum Policy Task Force, Report EI Docket no. 02-135 (Federal Communications Commission, Washington D.C, 2002)

4. J Mitola, GQ Maguire Jr, Cognitive radio: making software radios more personal. IEEE Pers. Comm. 6(4), 13-18 (1999)

5. OFCOM, Digital dividend: cognitive access, Statement on licence-exempting cognitive devices using interleaved spectrum (Office of Communications, London, 2009)

6. TYucek, H Arslan, A survey of spectrum sensing algorithms for cognitive radio applications. IEEE Communications Survey and Tutorials 11(1), 116-130 (2009)

7. D Cabric, SM Mishra, RW Brodersen, Implementation issues in spectrum sensing for cognitive radios, in Proceedings of Asilomar Conference on Signals, Systems and Computers, Pacific Grove, 7-10 Nov 2004, pp. 772-776

8. G Ganesan, YG Li, Agility improvement through cooperation diversity in cognitive radio, in Proceedings of IEEE GLOBECOM, St. Louis, 2 Dec 2005, pp. 2505-2509

9. A Ghasemi, ES Sousa, Collaborative spectrum sensing for opportunistic access in fading environments, in Proceedings of the 1st IEEE Symposium on New Frontiers in Dynamic Spectrum Access Networks, Baltimore, 8-11 Nov 2005, pp. 131-136
10. IF Akyildiz, BF Lo, R Balakrishnan, Cooperative spectrum sensing in cognitive radio networks: a survey. Phys. Commun. 4(1), 40-62 (2011)

11. Y-J Choi, Y Xin, S Rangarajan, Overhead-throughput tradeoff in cooperative cognitive radio networks, in Proceedings of the IEEE Wireless Communications and Networking Conference 2009, Budapest, 5-8 Apr 2009, pp. 1-6

12. S Maleki, SP Chepuri, G Leus, Energy and throughput efficient strategies for cooperative spectrum sensing in cognitive radios, in Proceedings of the 12th IEEE International Workshop on Signal Processing Advances in Wireless Communications 2011, San Francisco, 26-29 Jun 2011, pp. 71-75

13. S Maleki, SP Chepuri, G Leus, Optimization of hard fusion based spectrum sensing for energy-constrained cognitive radio networks. Physical Communication (2012). doi:10.1016/j.phycom.2012.07.003

14. L Chia-han, W Wolf, Energy efficient techniques for cooperative spectrum sensing in cognitive radios, in Proceedings of the 5th IEEE Consumer Communications and Networking Conference 2008, Las Vegas, 10-12 Jan 2008, pp. 968-972

15. A Malady, C da Silva, Clustering methods for distributed spectrum sensing in cognitive radio systems, in Proceedings of IEEE Military Communications Conference, San Diego, 16-19 Nov 2008, pp. 1-5

16. Y Selen, H Tullberg, J Kronander, Sensor selection for cooperative spectrum sensing, in Proceedings of the 3rd IEEE Symposium on New Frontiers in Dynamic Spectrum Access Networks, Chicago, 14-17 Oct 2008, pp. 1-11

17. G Ding, Q Wu, F Song, J Wang, Decentralized sensor selection for cooperative spectrum sensing based on unsupervised learning, in Proceedings of IEEE International Conference on Communications, Ottawa, 10-15 Jun 2012, pp. 1576-1580

18. J Lunden, V Koivunen, A Huttunen, HV Poor, Censoring for collaborative spectrum sensing in cognitive radios, in Proceedings of the 41st Asilomar Conference on Signals, Systems and Computers, Pacific Grove, 4-7 Nov 2007, pp. $772-776$

19. C Sun, W Zhang, KB Letaief, Cooperative spectrum sensing for cognitive radios under bandwidth constraints, in Proceedings of IEEE Wireless Communications and Networking Conference, Hong Kong, 11-15 Mar 2007, pp. 1-5

20. E Axell, G Leus, E Larsson, Overview of spectrum sensing for cognitive radio, in Proceedings of the 2nd International Workshop on Cognitive Information Processing, Elba Island, 14-16 Jun 2010, pp. 322-327

21. S Maleki, G Leus, Censored truncated sequential spectrum sensing for cognitive radio networks, in Proceedings of the 17th International Conference on Digital Signal Processing, Corfu, 6-8 Jul 2011, pp. 1-8

22. S Maleki, A Pandharipande, G Leus, Energy-efficient distributed spectrum sensing for cognitive sensor networks. IEEE Sens. J. 11(3), 565-573 (2011)

23. PK Varshney, Distributed Detection and Data Fusion (Springer, New York, 1997)

24. J Unnikrishnan, W Veeravalli, Cooperative sensing for primary detection in cognitive radio. IEEE J. Sel. Top. Signal Proc. 2(1), 18-27 (2008)

25. J Ma, G Zhao, Y Li, Soft combination and detection for cooperative spectrum sensing in cognitive radio networks. IEEE Trans. Wireless Comm. 7(11), 4502-4507 (2008)

26. $\mathrm{R}$ Wei Zhang, K Mallik, Optimization of cooperative spectrum sensing with energy detection in cognitive radio networks. IEEE Trans. Wireless Commun. 8(12), 5761-5766 (2009)

27. D Cabric, A Tkachenko, R Brodersen, Spectrum sensing measurements of pilot, energy, and collaborative detection, in Proceedings of the IEEE Military Communications Conference, Washington D.C., 23-25 Oct 2006, pp. 1-7

28. J Quaresma, C Ribeiro, A Gameiro, M Zelenak, J Duplicy, Distributed RF sensing framework with radio environment emulation, in Proceedings of the 2nd IEEE International Workshop on Emerging Cognitive Radio Applications and Algorithms, Madrid, 4-7 Jun 2013, pp. 1-6

29. GNU Radio, (2011). http://gnuradio.org/redmine/projects/gnuradio/wiki. Accessed 17 Jun 2013

30. USRP - Universal Software Radio Peripheral,? (2013). http://www.ettus.com Accessed 17 Jun 2013

31. Agilent Technologies, (2013). http://www.agilent.com. Accessed 17 Jun 2013

32. 3rd Generation Partnership Project, Evolved universal terrestrial radio access (E-UTRA); user equipment (UE) radio transmission and reception (release 8), Technical Report TS 36.803, V1.1.0 (3rd Generation Partnership Project, Sophia-Antipolis, 2008)

doi:10.1186/1687-1499-2013-191

Cite this article as: Gameiro et al: Selective reporting - a half signalling load algorithm for distributed sensing. EURASIP Journal on Wireless Communications and Networking 2013 2013:191. 